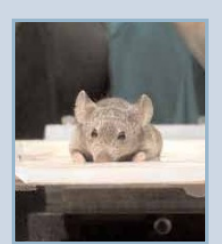

p882 Patent pare-down: European policy opens up research options

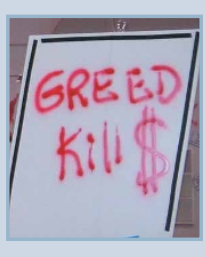

p884 Costly concerns: Pharma forced to create new solutions for drug pricing. p886 Flu fighter:

Malik Peiris takes

on SARS and

avian influenza.

\title{
Edible vaccines not ready for main course
}

Edible vaccines produced in genetically modified crops promise cheap and effective protection against infectious diseases in the developing world. But vaccine manufacturers are reluctant to take a bite out of the projects.

Since 1992, when biologist Charles Arntzen proposed genetically modifying bananas to serve as cheap vaccines against infectious diseases, research on plant-based pharmaceuticals has grown rapidly. In July, the European Union promised $€ 12$ million to European and South African scientists developing vaccines or antibodies against HIV/AIDS, rabies and tuberculosis. Work is further ahead in the US, where several acres of crops, most of them still experimental, are planted each year.

Researchers have thus far produced more than 45 different antigens in a wide range of plants. "In 10-15 years," says Hilary Koprowski, a veteran vaccine researcher at Thomas Jefferson University, "plant-derived vaccines will be fully appreciated."

At least in theory, plant-based vaccines would be safer than those produced in animal tissues because the chances of unknown human pathogens hitching a ride would be extremely small. Costs per dose would be low, and scaling up would just mean planting a larger crop. During transport and storage, vaccine-containing seeds or dried leaves would not need refrigeration, a significant advantage in developing countries. Oral vaccines especially, administered as juices or tablets to circumvent dose variability, would pave the way for mass vaccinations in those countries.

But even Arntzen now says his original idea of distributing vaccine-bearing fruit was naive, because regulatory agencies will not approve vaccines with variable dosing.

Many in the field say that, at least in animals, plant-based oral vaccines have been proven to be safe and effective. For instance, corn loaded with proteins from a gastroenteritis virus is effective, at least when used as a booster, in protecting pigs against the disease, says John Howard, founder of the Texas-based firm ProdiGene.

In humans, several vaccines have passed safety trials. Arntzen's group at Arizona State University tried vaccines produced in genetically modified potatoes and corn against enterotoxic Escherichia coli and Norwalk virus. Koprowski's group fed volunteers spinach containing a rabies booster vaccine. Both groups have tested oral hepatitis B vaccines, either as primary or booster vaccine, in lettuce, spinach and potatoes.

Although small phase 1 trials like these can't prove protection, volunteers in the studies showed an "appropriate," though not always strong, immune response, Arntzen says.

Arntzen is collaborating with companies in Egypt, South Africa and South Korea, but outside developing nations, where there is an urgent need for such vaccines, finding manufacturers willing to finance larger trials to demonstrate efficacy has been a formidable challenge. "I've talked to all of the [big companies]," says Koprowski, "and so far I regard it a waste of time."

Vaccine manufacturers have little reason to replace existing production lines, as most vaccines are economically unattractive. The medical community is also focused on high-tech approaches, making farm-grown vaccines a tough sell, Koprowski says. But smaller companies, led by young people willing to take risks, could challenge the current thinking, he says. "Then, others will follow."

Part of the hesitation stems from the fact that plant-based oral vaccines constitute a new technology from both a regulatory and scientific perspective, says renowned

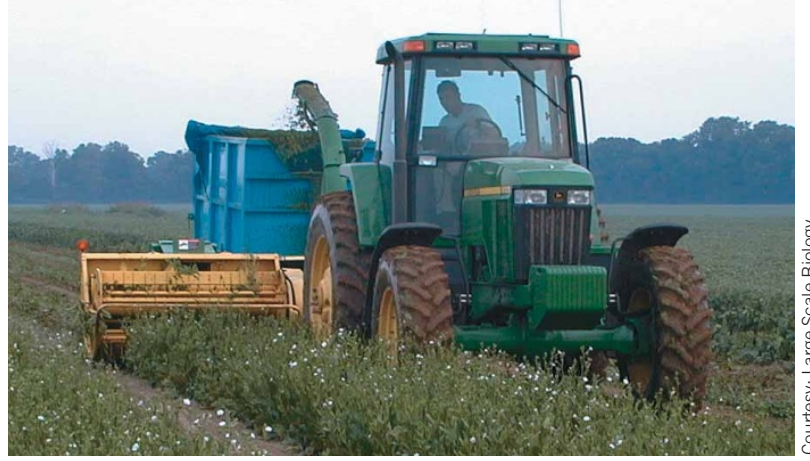

Food for thought: Plant-based vaccines not yet ripe enough for harvesting. vaccinologist Stanley Plotkin, who now advises Aventis Pasteur.

Before they can be approved, Plotkin says, plant-based vaccines will have to consistently generate stronger immune responses, which would need to be studied carefully for every crop. "If vaccines are intimately presented together with food, the gut's immune system faces a conundrum," he notes. The gut is designed not to react to antigens in food, but must produce a useful response against the vaccine. Instead of being immunized, patients could even end up being 'tolerized,' meaning an immune response against future invaders would be weakened, not intensified.

Researchers say they have not yet seen signs of such tolerance, but Plotkin says experiments to convince regulators have yet to be designed. "Immunologists will have to figure out how the gut can do this, and do it right $99.9999 \%$ of the time," he says. Producing veterinary vaccines first, followed by human booster vaccines, could be the sensible way forward, he adds.

Convincing the general public that it is safe to grow vaccines in fields poses a bigger challenge. Citing fears over supermarket shelves stocked with vaccine-contaminated foods, consumer groups have called for a ban on using food crops to produce pharmaceuticals. Some companies are avoiding the issue by developing injectable plant-based vaccines, by using nonfood crops or by not using genetically modified crops.

For instance, California-based Large Scale Biology uses genetically engineered mosaic viruses to infect tobacco plants. A few weeks later, says Larry Grill, the company's chief scientific officer, antibodies can be purified from the harvested leaves. The company has produced patientspecific antibodies against cancer cells just months after biopsies were taken.

Once the scientific and regulatory hurdles are cleared, convincing skeptics will be easier, researchers note. "If I could save millions of lives in developing countries," says Arntzen, "I think I'd have a pressure group that could stand up even against Greenpeace in Europe."

Peter Vermij, Amsterdam 